\title{
IncRNA GCInc1 may contribute to the progression of ovarian cancer by regulating p53 signaling pathway
}

\author{
Hu Li, ${ }^{1,2}$ Zheng Zeng, ${ }^{3}$ Xiang Yang, ${ }^{1}$ Ye Chen, ${ }^{1}$ Lei He, ${ }^{4}$ Ting Wan ${ }^{5}$ \\ ${ }^{1}$ Department of Gynecology, Panyu Central Hospital, Guangzhou \\ ${ }^{2}$ Cancer Institute of Panyu Central Hospital, Guangzhou \\ ${ }^{3}$ Department of Gynecology, Third Affiliated Hospital of Sun Yat-sen University, Guangzhou \\ ${ }^{4}$ Sun Yat-sen Memorial Hospital, Sun Yat-sen University, Guangzhou \\ ${ }^{5}$ Sun Yat-Sen University Cancer Center, State Key Laboratory of Oncology in South China, Collaborative Innovation \\ Center for Cancer Medicine, Guangzhou, China
}

\begin{abstract}
Ovarian cancer (OC) is one of the most prevalent and deadly types of gynecological malignancy. Since current treatments are not effective against $\mathrm{OC}$, it is imperative to develop novel potential therapeutic targets for managing OC. In this study, we aimed to uncover the underlying molecular mechanism of long non-coding RNA (lncRNA) GClnc1 related to p53 signaling pathway in OC. The expression of lncRNA H19 GClnc1 was markedly higher in OC samples than the related normal tissues. Next, we found that lncRNA GClnc1 inhibited p53. In addition, the lncRNA GClnc1 overexpression promoted the cell proliferation and migration in vitro. Subsequently, p53 silencing obligated the effect of lncRNA GCln1 knock down on cell proliferation and migration. To sum up, lncRNA GClnc1 contributes to the progression of OC by regulating p53 signaling pathway. Meanwhile, our findings also suggested that lncRNA GClnc1 may serve as a novel therapeutic target for OC patients.
\end{abstract}

Key words: IncRNA GClnc1; p53; ovarian cancer; target therapy.

Correspondence: Dr. Lei He, Sun Yat-sen Memorial Hospital, Sun Yat-sen University, Guangzhou, China. E-mail: helei33@mail.sysu.edu.cn

Contributions: HL, ZZ, contributed equally to this study. LH, TW, study conception; HL, ZZ, experiments design and completion, manuscript drafting; XY, YC, sample collection, contribution to data analysis. All the authors have read and approved the final version of the manuscript and agreed to be accountable for all aspects of the work.

Funding: This research was funded by startup project of clinical medicine in Guangzhou (20171A010334) and by Science and Technology Planning Project of Panyu in Guangzhou (2017-Z04-20).

Conflict of interest: The authors declare that they have no competing interests, and all authors confirm accuracy.

Availability of data and materials: The data used to support the findings of this study are available from the corresponding author upon request.

Ethical Approval: All procedures with human subjects in this study were conducted in accordance with the Human Ethics Committee of the Sun Yat-sen Memorial Hospital of Sun Yat-sen University, China.

Patient consent for publication: All subjects gave their informed consent for inclusion before they participated in the study. 


\section{Introduction}

Ovarian cancer (OC) is one of the common tumors of the female reproductive system and leading causes of death among females. ${ }^{1,2}$ Current treatments are not effective against OC, the overall survival of OC patients is still unsatisfactory. The five year survival rate of around $30 \%$ with advances in surgery and chemotherapy. ${ }^{3}$ Thus, it is imperative to develop novel potential therapeutic targets for managing OC.

Long non-coding RNAs (lncRNAs) belongs to the non-coding RNAs family with more than 200 nucleotides in length. Although IncRNAs do not template protein synthesis, they can regulate gene expression at transcriptional or post-transcriptional level. ${ }^{4}$ lncRNAs have been reported to play vital roles in regulating cellular processes, such as signaling transduction, in various cancers. ${ }^{5}$ Currently, it has been found that lncRNAs plays important roles in the progression of OC. ${ }^{6}$ However, the specific influence of lncRNA GGlnc1 on the progression of OC remains largely unclear.

The transcription factor $\mathrm{p} 53$ is $\sim 16-20 \mathrm{~kb}$ in length, is a pair of alleles and acts as a tumor suppressor gene localized on human chromosome 17. ${ }^{7}$ P53 functions as a suppressor of cell growth, and alterations in $\mathrm{p} 53$ lead to loss of this negative growth regulation and more rapid cell proliferation. The dysfunction of p53 contributes to the development of most human cancers. ${ }^{8}$ Recently, lncRNAs have been shown to interact with p53 to play regulating roles in various cancers. ${ }^{9}$ However, the interaction of IncRNA GGlnc1 and p53 on the progression of OC still remains unknown.

In this study, we aim to uncover whether lncRNA GClnc1 could regulate the progression of OC via p53 signaling pathway.

\section{Materials and Methods}

\section{Clinical samples collection}

Human OC tissue samples as well as related non-tumorous tissues were obtained from 42 patients, and all the specimens were reviewed and verified by pathologists and immediately frozen in liquid nitrogen. All subjects gave their informed consent for inclusion before they participated in the study. All experimental protocols were approved by the Ethics Committee.

\section{RNA in situ hybridization}

The RNA in situ hybridization (ISH) was performed as previously described. ${ }^{9}$ The in situ detection of GClnc1 was performed on 6- $\mu \mathrm{m}$ formalin-fixed, paraffin-embedded sections using DIGlabeled miRCURYTM Detection probe (Exiqon, Woburn, MA, USA). Nikon 80i microscope with Nikon NIS-Elements F 2.3 software (Nikon, Shanghai, China) were used to analyze.

\section{Cell culture}

The human OC cell line, ES-2, was purchases from ATCC
(Rockville, MD, USA). Cells were maintained in RPMI 1640 (Gibco, Grand Island, NY, USA). The normal immortalized human ovarian surface epithelial cell line T1074 was obtained from Abcam (Shanghai, China) and cultured in Prigrow 1 medium (Abcam, Shanghai, China). Medium was plus $10 \%$ fetal bovine serum (FBS, HyClone, Logan, UT, USA), and 1\% streptomycin and penicillin (Sigma-Aldrich, St. Louis, MO, USA), and the cells were grown at $37^{\circ} \mathrm{C}$ with $5 \% \mathrm{CO}_{2}$.

\section{qRT-PCR}

TRIzol Plus RNA purification system was used to extract total RNA from specimens (Thermo Fisher Scientific, Beijing, China). Residual DNA was removed using DNA-free DNase (Ambion, Austin, TX). The cDNA was generated by reverse transcription using Moloney murine leukemia virus reverse transcriptase (Invitrogen, Beijing, China). The SYBR Green method was performed to detect the expression of lncRNA GClnc1, p21, p53 and GAPDH. The primer sequences were listed in Table 1.

\section{Overexpression and suppression constructs}

The si-GClnc1, si-p53, pcDNA-GClnc1 and their related negative controls (si-NC and pcDNA-NC) were purchased from Suzhou Hongxun Biotechnologies (Suzhou, China). These constructs were transfected when cell density reached $60 \%$ according to the manufacturer's instructions of Lipofectamine 2000 (Invitrogen). The culture medium was replaced after $6 \mathrm{~h}$.

\section{Cell counting kit-8 (CCK-8) assay}

CCK-8 assay kit was purchased from Dojindo (Shanghai, China) to monitor the cell viability. Cells were seeded in the 96well plate (BD Biosciences, Shanghai, China) at a density of 5000 cells per well. After incubation for indicated time $(0,6,24,48,72$ and $96 \mathrm{~h}$ ), we added $10 \mu \mathrm{L} \mathrm{CCK}-8$ reagent into each well at indicated time point followed by incubation for $1 \mathrm{~h}$ at $37^{\circ} \mathrm{C}$. Subsequently, the optical density (OD) was measured at $450 \mathrm{~nm}$ on a microplate reader.

\section{Transwell assay}

Cell migration was assayed by Boyden chamber assay with 24 well transwell permeable supports with $8 \mu \mathrm{m}$ pores (Corning Coaster, Lowell, MA, USA). $200 \mu \mathrm{L}$ of serum-free medium containing $0.1 \mathrm{M}$ cells for the migration assay were added to the filter. The bottom chamber was prepared with $750 \mu \mathrm{L}$ complete cell culture medium in which the FBS as a chemoattractant. After incubated for indicated time, the non-invasive cells were cleaned by scrubbing with a cotton swab. The cells that adhered to the outside of the membrane were fixed and dyed with crystal violet solution. The stained cells were dissolved in extraction buffer and solutions were transferred to a 96-well culture plates for colorimetric reading at OD $560 \mathrm{~nm}$. The OD values reflected the cell ability of migration or invasion. ${ }^{10}$

\section{Western blotting}

The protein expression levels of p53, p21 and BAX after diffe-

Table 1. List of the primers used.

\begin{tabular}{lll} 
& Forward primer & Reverse primer \\
lncRNA GClncl & TGGGGTAACTTAGCAGTTTCAAT & GGCAAGCAGTAATCTTACATGACAC \\
p53 & CCCAAGCAATGGATGATTTGA & ATGAGGGTGCTGTCTTTGTAGG \\
\hline p21 & GGCAGACCAGCATGACAGATT & GCGGATTAGaGGCTTCCTCT \\
\hline
\end{tabular}


rent treatment was detected by Western blotting as described previously. ${ }^{11}$ The primary antibodies: anti-p53 (1:500, Millipore, Bedford, MA, USA), anti-p21 (1:500, Millipore), and GAPDH (Millipore). The secondary antibody was the goat anti-rabbit horseradish peroxidase (HRP)-conjugated antibody (1:2,000; BioRad, Philadelphia, PA, USA). All the results are from separate blots.

\section{Dual luciferase reporter assay}

Cells were inoculated into the 24 -well plate $\left(3 \times 10^{5}\right.$ cells / well). Then, the cells were co-transfected with wild-type or mutant psiCHECK-2 p53 vector (Generay, Shanghai, China) and pcDNAGClnc1 or pcDNA-NC with Lipofectamine 2000 (Thermo Fisher Scientific, Beijing, China) according with the manufacture's instruction. The luciferase activity of cells was measured by a Dual-Luciferase Reporter assay kit (Promega, Shanghai, China) after $24 \mathrm{~h}$.

\section{RNA-binding protein immunoprecipitation analysis}

RNA immunoprecipitation (RIP) assay was used to detect the association of p53 and lncRNA GClnc1 according to the manufacturer's instructions of the Magna RIP RNA Binding Protein Immunoprecipitation Kit (Sigma-Aldrich). Finally, RNA was dissolved in $10 \mu \mathrm{L}$ of diethyl pyrocarbonate (DEPC) water (Beyotime, Shanghai, China), and stored at $-80^{\circ} \mathrm{C}$. Subsequently, qRT-PCR was used to determine the expression of GClnc1 in cop53 protein and $\mathrm{IgG}$ protein precipitate.

\section{Statistical analysis}

Statistical Product and Service Solutions (SPSS, Chicago, IL, USA) 16.0 statistical software was used for all statistical analysis Results are shown as means $\pm \mathrm{SE}$ (standard errors). The unpaired student's t-test were used to compare the significance of differences between the mean of different groups. A value of $p<0.05$ indicated the statistical significance.

\section{Results}

\section{IncRNA GCInc1 is enriched in OC tissues}

lncRNA GClnc1 has been found to be upregulated and play important roles in bladder cancer and colorectal cancer. ${ }^{12}$ ISH (Figure 1A) was used to establish the pathologic and clinical significance of GClnc1 expression in OC, which was carried out by the pathology department. To explore the relationship between the expression of GClnc1 and the development of OC, we also measured the expression levels of lncRNA GClnc1 in 18 OC tissue samples and related non-tumorous tissues using qRT-PCR. As shown in Figure 1B, the expression levels of lncRNA GClnc1 in OC cancer tissues were conspicuously higher than the related normal tissues. It indicated that GClnc1 was involved in the progression of OC.

\section{IncRNA GCInc1 expression level alters cell prolifera- tion and migration in vitro}

To elucidate the underlying molecular mechanism of lncRNA GClnc1 regulating the progress of $\mathrm{OC}$, we subsequently measured the expression of GClnc1 in ES-2 and T1074 cells. As shown in Figure 2A, GClnc1 expression in ES-2 cells was markedly higher than that of T1074 cells by qRT-PCR. In order to investigate the impact of GClnc1 on cell proliferation of OC cells, we knocked down GClnc1 by si-GClnc1. The expression level of GClnc1 was obviously decreased in ES-2 cells compared with si-NC, the negative control (Figure 2B). The cell proliferation and migration were significantly decreased after knockdown of GClnc1 expression by CCK-8 and transwell assays (Figure 2 C,D). Next, we overexpressed GClnc1 in T1074 cells. The expression level of GClnc1 was markedly increased in T1074 cells compared with pcDNA-NC (Figure 2E). Similarly, the cell proliferation and migration were significantly increased after overexpression of GClnc1 by CCK-8 and transwell assays (Figure 2 F,G).

\section{IncRNA GCInc1 inhibits p53 activity}

To illustrate the underlying mechanism of GClnc1 regulating the progression of $\mathrm{OC}$, we further investigate the interaction between GClnc1 and p53. The level of GClnc1 in the p53 antibody

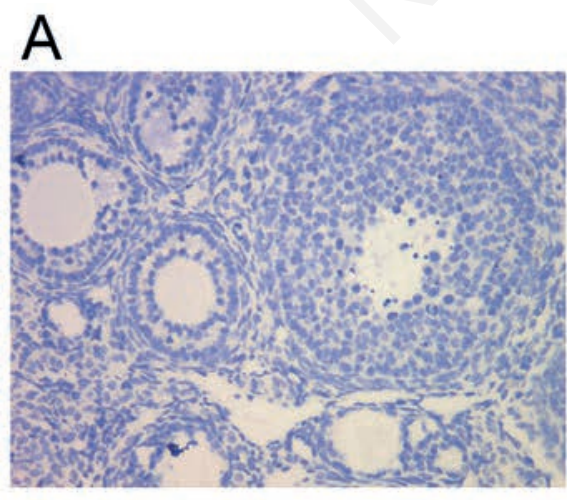

Non-tumorous tissues
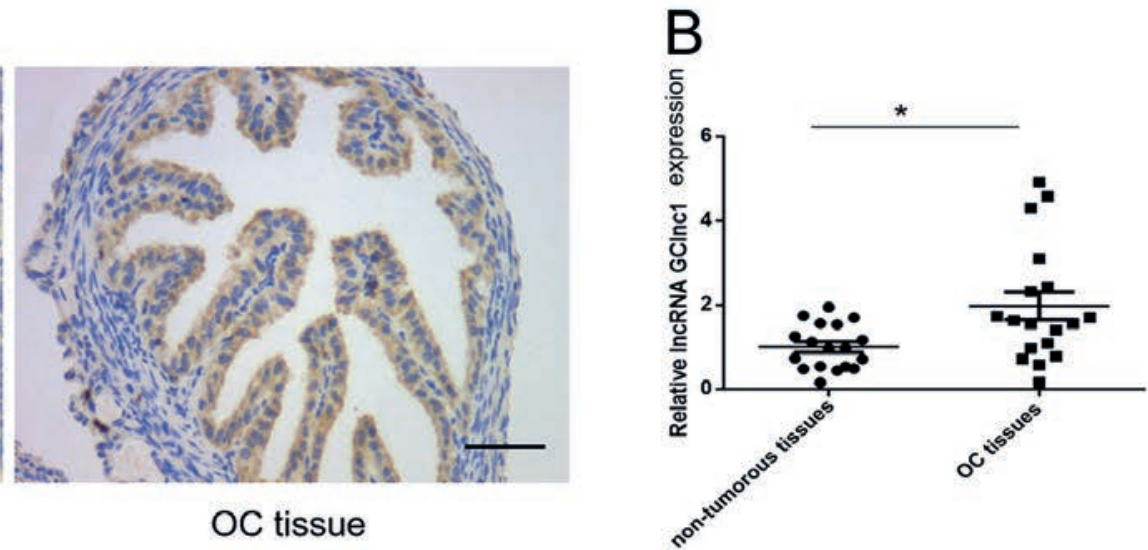

Figure 1. The expression of lncRNA GClnc1 in $18 \mathrm{OC}$ patients was significantly higher than that of non-tumorous tissues. A) Representative images of GClnc1 expression in OC tissue and non-tumorous tissues were evaluated by ISH; scale bar: $100 \mu \mathrm{m}$ ). B) GClncl expression in OC tissue and non-tumorous tissues was measured by $\mathrm{qRT}-\mathrm{PCR} .{ }^{*} \mathrm{p}<0.05$ vs non-tumorous tissues. 
A

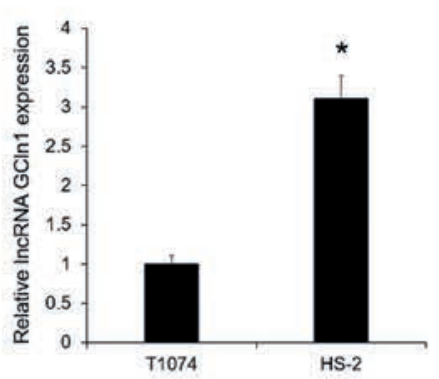

B

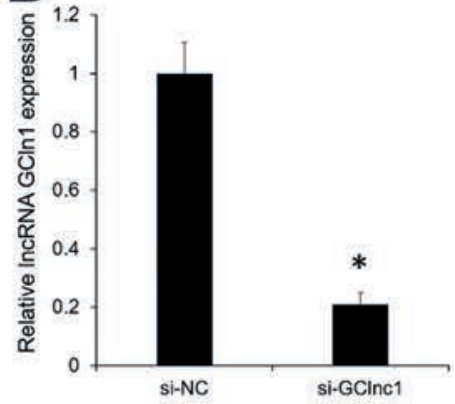

C

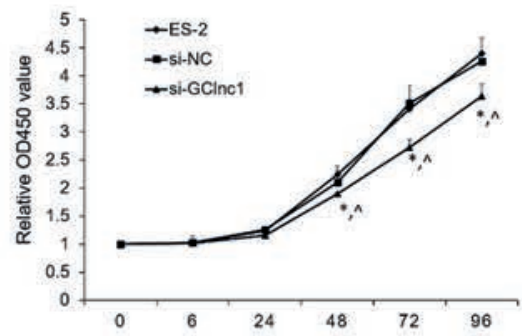

D

si-NC

si-GClnc1

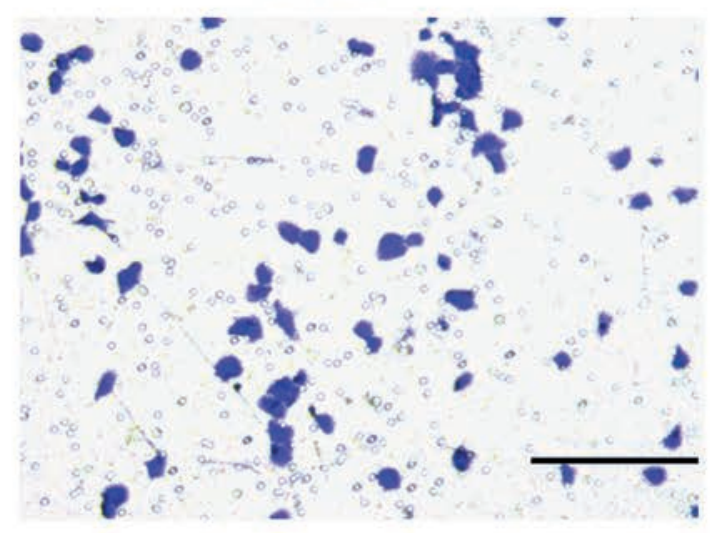

E

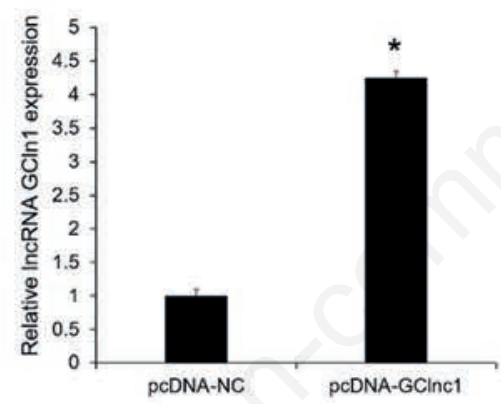

F

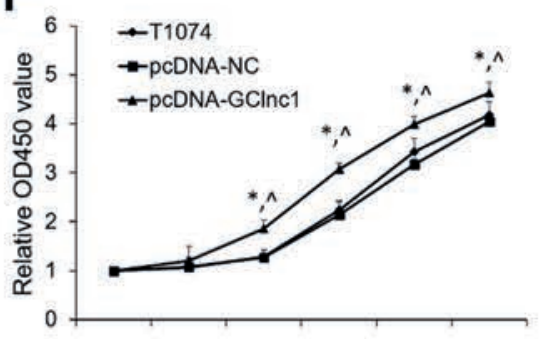

G
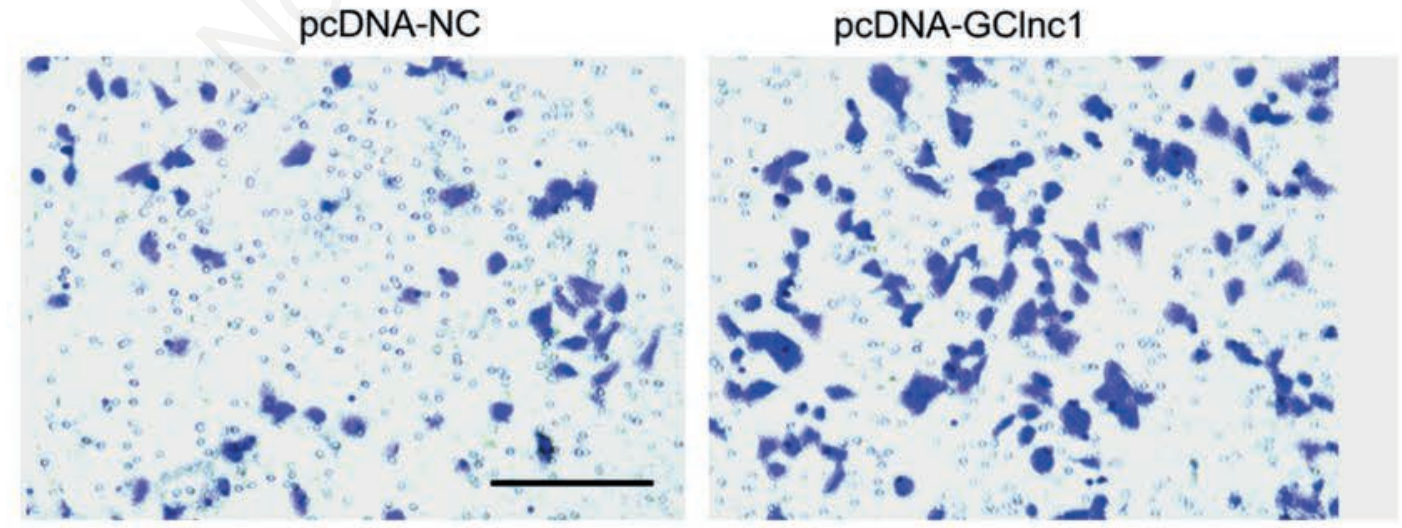

Figure 2. IncRNA GClnc1 expression altered the cell proliferation and migration in vitro. A) GClnc1 expression in ES-2 cells was significantly higher compared with T1074 cell by qRT-PCR. B) GClnc1 expression was remarkedly decreased in ES-2 cells after knock down. C) GClnc1 knockdown significantly suppressed the proliferation of ES-2 cells. D) GClnc1 knockdown significantly decreased the migration of ES-2 cells; scale bar: $10 \mu \mathrm{m}$. E) GClnc1 expression was markedly increased in ES-2 cells after overexpression. F) GClnc1 overexpression significantly promoted the proliferation of T1074 cells. G) GClnc1 overexpression significantly increased the migration of T1074 cells; scale bar: $10 \mu \mathrm{m} .{ }^{*} \mathrm{p}<0.05 v s$ si-NC or pcDNA-NC; ${ }^{\wedge} \mathrm{p}<0.05$ vs $\mathrm{ES}-2$ or T1074 $(\mathrm{n}=3)$. 
precipitation complex was found to be significantly higher than that of the IgG control group via RIP experiment in ES-2 cells (Figure 3A). Next, the luciferase reporter gene assay proved that overexpression of GClnc1 inhibited the luciferase activity of p53 in T1074 cells (Figure 3B). Furthermore, the mRNA and protein expression of $\mathrm{p} 53$, as well as $\mathrm{p} 21$, were markedly decreased after GClnc1 overexpression (Figure 3 C,D). Those data suggested that GClnc1 inhibited the activity of p53.

IncRNA GCInc1 promotes cell proliferation and migration via $\mathrm{p53}$

To verify that GClnc1 promoted the proliferation of OC cells by altering p53 activity, silenced GClnc1 was knocked down in ES-2 cells. At the same time, p53 was simultaneously silenced in cells as well (Figure 4A). The CCK-8 and transwell experiments showed that p53 silencing markedly reversed the inhibition in cell proliferation and migration caused by GClnc1 knockdown (Figure 4 B,C).

\section{Discussion}

Molecular mechanisms underlying the progression of OC still remain complex and largely unknown. In this study, we mainly investigated the biological function of lncRNA GClnc1 and p53 interaction in the progression of OC.

Numerous studies have reported that lncRNAs are regulators in a wide range of biological functions and play complex and extensive roles in cancer development and progression. ${ }^{13,14}$ Among those widely studied lncRNAs, lncRNA GClnc1 has attracted a lot of attention with targeting multiple genes, such as MYC. IncRNA GClnc1 has been reported to promote proliferation and invasion of bladder cancer through activation of MYC. ${ }^{12}$ Meanwhile, IncRNA GClnc1 has been identified to promote gastric carcinogenesis and may act as a modular scaffold of WDR5 and KAT2A complexes to specify the histone modification pattern. ${ }^{15}$ In our work, GClnc1 was found to be remarkedly upregulated in OC tissue samples compared with related normal tissues. What's more, the overex-
A
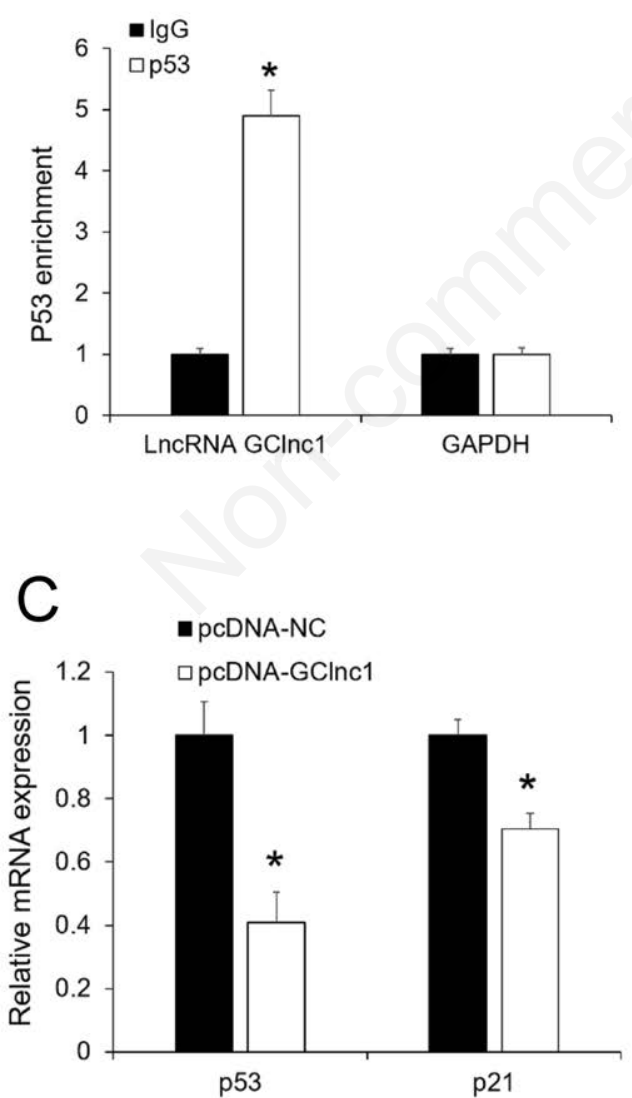

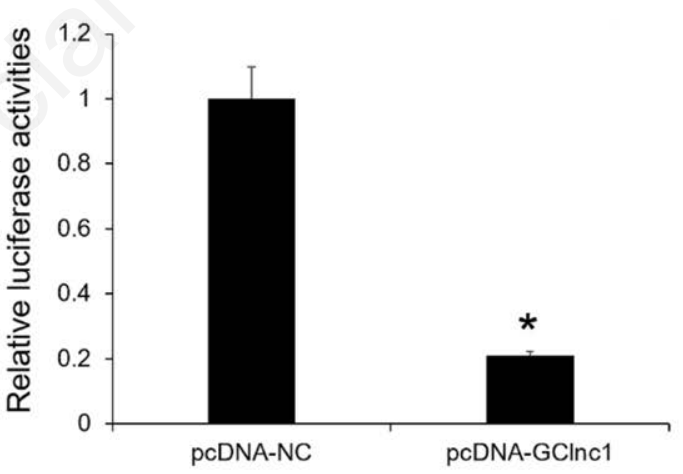

p53

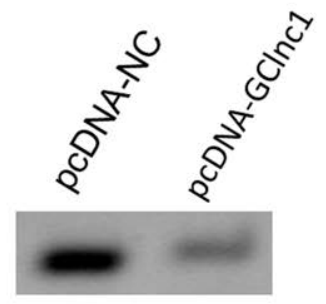

p21

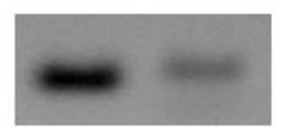

GAPDH

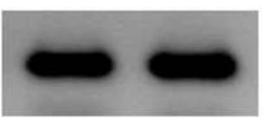

Figure 3. IncRNA GClnc1 interacted with p53. A) The correlation of GClncl and p53 was detected by RIP and qRT-PCR. B) Luciferase reporter assay was used to investigate the activity of p53 after GClnc1 overexpression. C) p53 and p21 expression levels were analyzed by $\mathrm{qRT}$-PCR. D) Western blotting analysis was performed to determine the protein expression levels of $\mathrm{p} 53$ and $\mathrm{p} 21 .{ }^{*} \mathrm{p}<0.05 v s$ negative control $(n=3)$. 

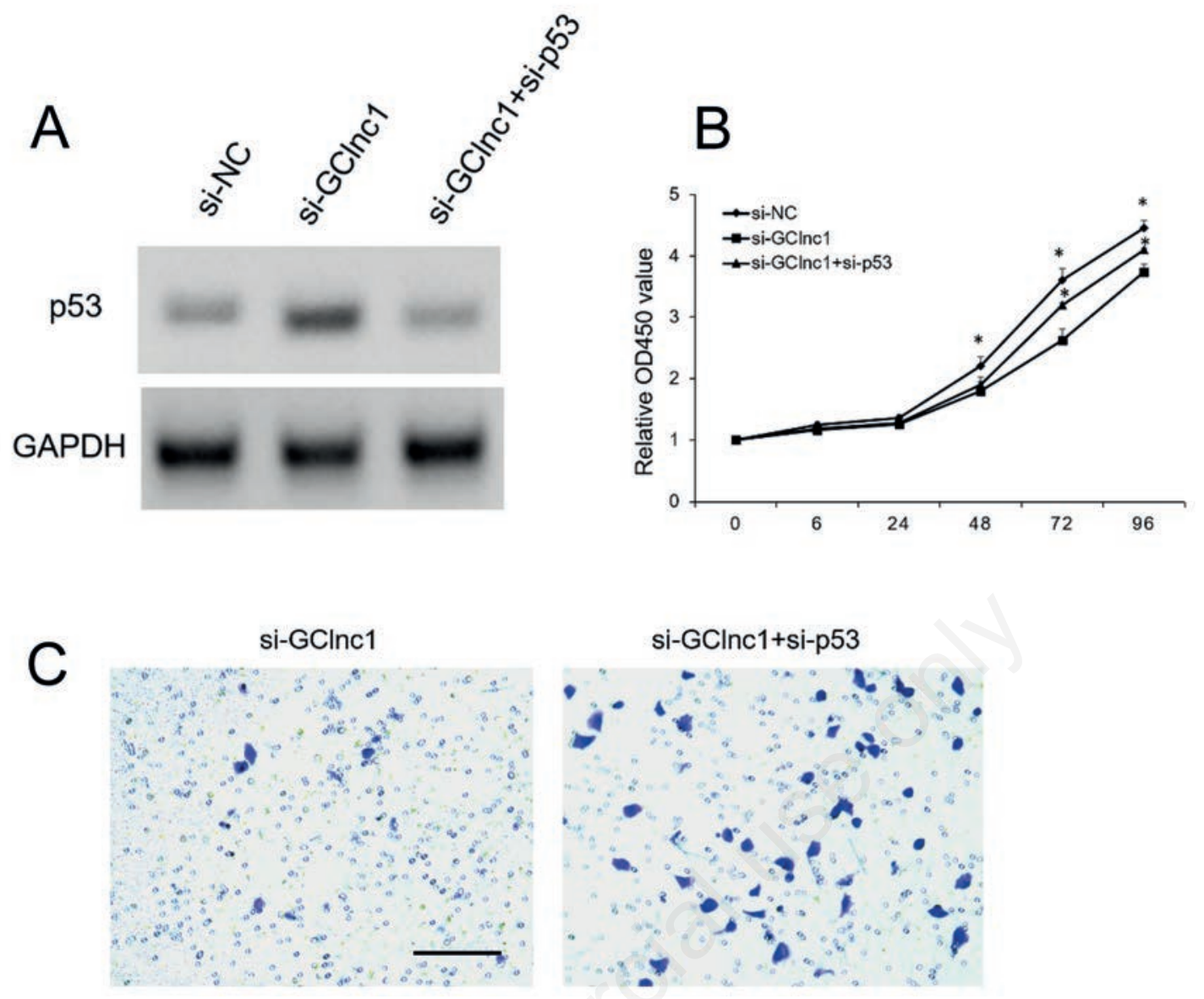

Figure 4. IncRNA GClnc1 altered cell proliferation and migration through p53. A) Western blotting was carried out to determine the protein expression level of p53 in cells with p53 silencing after knockdown of GClnc1. B) p53 silencing obligated the suppression of cell proliferation caused by knockdown of GClnc1. C) p 53 silencing reversed the inhibition of cell migration caused by knockdown of GClnc1; scale bar: $10 \mu \mathrm{m} .{ }^{*} \mathrm{p}<0.05$ vs si-GClnc1 ( $\left.\mathrm{n}=3\right)$.

pression of GClnc1 was also confirmed to promote the cell proliferation and migration, while the knockdown of GClnc1 was verified to suppress the cell proliferation and migration in vitro.

Recently, accumulated studies have shown that p53 signaling pathway interacted with lncRNA GClnc1 to play important roles in various cancers. IncRNA GClnc1 has been confirmed to promote the progression of colorectal cancer by inhibiting p53 signaling pathway. ${ }^{9}$ In addition, lncRNA GClnc1 has also been reported to promote tumorigenesis in osteosarcoma by inhibiting p53 signaling. ${ }^{16}$ In our work, we found that $\operatorname{lncRNA}$ GCln1 inhibit p53, as well as p21, activity in OC. Moreover, the silencing of p53 reversed the effect of GClnc1 knockdown on the proliferation of the human OC cell line, ES-2. These results allow suggesting that lncRNA GClnc1 may contribute to the progression of OC by regulating p53 signaling pathway. However, to confirm this hypothesis, further studies should be performed in vivo and on cell models in vitro to directly assess whether lncRNA GClnc1 might induce possible alternation in the proliferation related biomarkers and the dysfunction of $\mathrm{p} 53$ downstream genes in OC cells. Once its role in modulating the p53 pathway had been confirmed, GClnc1 would become a novel therapeutic target for OC patients.

Based on our study results, we conclude the lncRNA GClnc1 contributes to the progression of OC by regulating p53 signaling pathway. Our findings suggested that GClnc1 may serve as a novel therapeutic target for OC patients.

\section{References}

1. Torre LA, Bray F, Siegel RL, Ferlay J, Lortet-Tieulent J, Jemal A. Global cancer statistics, 2012. CA Cancer J Clin 2015;65:87-108.

2. Yan HC, Fang LS, Xu J, Qiu YY, Lin XM, Huang HX, et al. The identification of the biological characteristics of human ovarian cancer stem cells. Eur Rev Med Pharmacol Sci 2014;18:3497-503.

3. Rustin G, van der Burg M, Griffin C, Qian W, Swart AM. Early versus delayed treatment of relapsed ovarian cancer. Lancet 2011;377:380-1.

4. Yang J, Wang X. Role of long non-coding RNAs in lymphoma: A systematic review and clinical perspectives. Crit Rev Oncol Hematol 2019;141:13-22.

5. Puvvula PK. LncRNAs regulatory networks in cellular senescence. Int J Mol Sci 2019;20:2615.

6. Chen ZJ, Zhang Z, Xie BB, Zhang HY. Clinical significance of up-regulated lncRNA NEAT1 in prognosis of ovarian cancer. 
Eur Rev Med Pharmacol Sci 2016;20:3373-7.

7. Mantovani F, Collavin L, Del Sal G. Mutant p53 as a guardian of the cancer cell. Cell Death Differ 2019;26:199-212.

8. Labuschagne CF, Zani F, Vousden KH. Control of metabolism by p53 - Cancer and beyond. Biochim Biophys Acta Rev Cancer 2018;187032-42.

9. Dong YX, Pang ZG, Zhang JC, Hu JQ, Wang LY. Long noncoding RNA GClnc1 promotes progression of colorectal cancer by inhibiting p53 signaling pathway. Eur Rev Med Pharmacol Sci 2019;23:5705-13.

10. Zhang J, Kong X, Li J, Luo Q, Li X, Shen L, et al. miR-96 promotes tumor proliferation and invasion by targeting RECK in breast cancer. Oncol Rep 2014;31:1357-63.

11. Yin Q, Fischer L, Noethling C, Schaefer WR. In vitro-assessment of putative antiprogestin activities of phytochemicals and synthetic UV absorbers in human endometrial Ishikawa cells. Gynecol Endocrinol 2015;31:578-81.
12. Zhuang C, Ma Q, Zhuang C, Ye J, Zhang F, Gui Y. LncRNA GClnc1 promotes proliferation and invasion of bladder cancer through activation of MYC. FASEB J 2019;33:11045-59.

13. Guo X, Gao L, Liao Q, Xiao H, Ma X, Yang X, et al. Long non-coding RNAs function annotation: a global prediction method based on bi-colored networks. Nucleic Acids Res 2013;41:e35.

14. Ponting CP, Oliver PL, Reik W. Evolution and functions of long noncoding RNAs. Cell 2009;136:629-41.

15. Sun TT, He J, Liang Q, Ren LL, Yan TT, Yu TC, et al. LncRNA GClnc1 promotes gastric carcinogenesis and may act as a modular scaffold of WDR5 and KAT2A complexes to specify the histone modification pattern. Cancer Discov 2016;6:784-801.

16. Sui Y, Han Y, Zhao X, Li D, Li G. Long non-coding RNA GClnc1 promotes tumorigenesis in osteosarcoma by inhibiting p53 signaling. Biochem Biophys Res Commun 2018;507:3642.

Received for publication: 23 July 2020. Accepted for publication: 4 September 2020.

This work is licensed under a Creative Commons Attribution-NonCommercial 4.0 International License (CC BY-NC 4.0).

CCopyright: the Author(s), 2020

Licensee PAGEPress, Italy

European Journal of Histochemistry 2020; 64:3166

doi:10.4081/ejh.2020.3166 